Prepared for the U.S. Department of Energy

under Contract DE-AC05-76RL01830

\title{
Reverse Osmosis Optimization
}

KL McMordie Stoughton

X Duan

EM Wendel

August 2013

Pacific Northwest

NATIONAL LABORATORY

Proudly Operated by Battelle Since 1965 


\title{
DISCLAIMER
}

This documentation was prepared as an account of work sponsored by an agency of the United States Government. Neither the United States Government nor any agency thereof, nor Battelle Memorial Institute, nor any of their employees, makes any warranty, express or implied, or assumes any legal liability or responsibility for the accuracy, completeness, or usefulness of any information, apparatus, product, or process disclosed, or represents that its use would not infringe privately owned rights. Reference herein to any specific commercial product, process, or service by trade name, trademark, manufacturer, or otherwise does not necessarily constitute or imply its endorsement, recommendation, or favoring by the United States Government or any agency thereof, or Battelle Memorial Institute. The views and opinions of authors expressed herein do not necessarily state or reflect those of the United States Government or any agency thereof.

\author{
PACIFIC NORTHWEST NATIONAL LABORATORY \\ operated by \\ BATTELLE \\ for the \\ UNITED STATES DEPARTMENT OF ENERGY \\ under Contract DE-AC05-76RL01830
}

Printed in the United States of America

Available to DOE and DOE contractors from the

Office of Scientific and Technical Information,

P.O. Box 62, Oak Ridge, TN 37831-0062;

ph: (865) 576-8401, fax: (865) 576-5728

email: reports@adonis.osti.gov

\begin{abstract}
Available to the public from the National Technical Information Service,
U.S. Department of Commerce, 5285 Port Royal Rd., Springfield, VA 22161

ph: (800) 553-6847, fax: (703) 605-6900

email: orders@ntis.fedworld.gov

online ordering: http://www.ntis.gov/ordering.htm
\end{abstract}

This document was printed on recycled paper.

$(8 / 00)$ 
PNNL-22682

\section{Reverse Osmosis Optimization}

KL McMordie Stoughton

$\mathrm{X}$ Duan

EM Wendel

August 2013

Prepared for U.S. Department of Energy under Contract DE-AC05-76RL01830

Pacific Northwest National Laboratory

Richland, Washington 99352 


\section{Contacts}

Will Lintner, P.E., CEM

Federal Energy Management Program

U.S. Department of Energy

1000 Independence Ave. SW

Washington, DC 20585-0121

Phone: (202) 586-3120

E-mail: william.lintner@ee.doe.gov

Kate McMordie Stoughton

Pacific Northwest National Laboratory

P.O. Box 999

Richland, WA

Phone: (888) 375-7665

E-mail: kate.mcmordie@pnnl.gov 


\section{Executive Summary}

This technology evaluation was prepared by Pacific Northwest National Laboratory on behalf of the U.S. Department of Energy's Federal Energy Management Program (FEMP). The technology evaluation assesses techniques for optimizing reverse osmosis (RO) systems to increase RO system performance and water efficiency. This evaluation provides a general description of RO systems, the influence of RO systems on water use, and key areas where RO systems can be optimized to reduce water and energy consumption. The evaluation is intended to help facility managers at Federal sites understand the basic concepts of the RO process and system optimization options, enabling them to make informed decisions during the system design process for either new projects or recommissioning of existing equipment. This evaluation is focused on commercial-sized RO systems generally treating more than 80 gallons per hour.

RO technology provides high purity water by forcing water through a semi-permeable membrane that filters out dissolved solids and other impurities. RO technology is used in applications where high purity water is essential including boiler feed water filtering, product rinsing, microelectronics production, laboratory testing, biotechnology, and other processes that require highly purified water.

This technology evaluation explores three categories of RO system optimization:

1. Feed water pretreatment: Dissolved and suspended solids can be filtered from water supplied to the RO membrane, which allows the membrane to operate more efficiently and enhances overall system performance.

2. Advanced and new membrane technologies: New membrane technology is commercially available that increases the membrane surface area and permeability to increase system performance, reducing both energy and water use.

3. Flow configuration: RO systems can be designed optimally to recirculate water through the system, reducing the amount of water supplied to the system.

There are several savings opportunities that can be achieved through optimizing RO systems including reduction in energy and water consumption and membrane maintenance and replacement costs. Wastewater treatment costs also may be reduced if there is a reduction in discharge to drain.

RO systems are energy intensive. But by optimizing the RO performance, the system can potentially have a lower operating pressure, resulting in less energy consumed. Optimizing RO systems also results in an increased water recovery rate, equating to a reduction in the total amount of feed water entering the system.

Another savings opportunity is membrane maintenance and replacement. Pretreatment optimization plays a key role in keeping the membrane clean and efficient, which reduces the frequency of membrane cleaning and extends the life of the membrane elements.

Because of the site specific nature of large RO systems, there is not a typical range of potential energy, water, and cost savings from system optimization. A detailed analysis for the particular installation will be required to estimate savings associate with system improvements. 
The evaluation includes two examples of optimized RO systems at Federal sites:

- Tobyhanna Army Depot recommissioned a large RO system to improve the system performance, which included feed water pretreatment, system configuration optimization, and pressure balancing. These improvements resulted in:

o Increased production rate of the system to $150 \%$ of design

o System recovery rate of $90 \%$

o Drop in system pressure requirements that doubled the flow rate through the system, reducing energy use

o Extension of the RO membrane life

o Decreased operation cost by over $\$ 90,000$ annually

- Sandia National Laboratories upgraded an existing RO system with a High Efficiency Reverse Osmosis $\left(\mathrm{HERO}^{\mathrm{TM}}\right.$ ) unit that provides highly purified rinsing water to a microelectronics processing complex. The HERO system provides specially designed pretreatment to the water before it enters the RO system, which increased the system recovery rate from $60 \%$ to $95 \%$, saving 34 million gallons annually. 


\section{Acknowledgements}

The authors of this report would like to thank the following individuals that provided support to the production of the technology evaluation.

Federal Energy Management Program:

Will Lintner

Pacific Northwest National Laboratory:

Shannon Colson

Brian Boyd

James Cabe

Jennifer Williamson

Matt Wilburn

Massine Merzouk

Susan Sande

Rose Zanders

Tobyhanna Army Depot:

Charles Valenza

Tom Wildoner 


\section{Abbreviations and Acronyms}

$\begin{array}{ll}\text { CA } & \text { cellulose acetate } \\ \text { FEMP } & \text { Federal Energy Management Program } \\ \text { gpm } & \text { gallon per minute } \\ \text { HERO } & \text { High Efficiency Reverse Osmosis } \\ \text { MESA } & \text { Microsystems and Engineering Sciences Applications } \\ \text { Mgal } & \text { million gallons } \\ \text { psi } & \text { pounds per square inch } \\ \text { RO } & \text { reverse osmosis } \\ \text { TDS } & \text { total dissolved solids } \\ \text { TFC } & \text { thin film composite } \\ \text { TSS } & \text { total suspended solids }\end{array}$




\section{Contents}

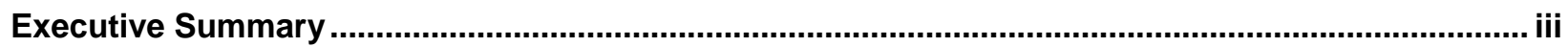

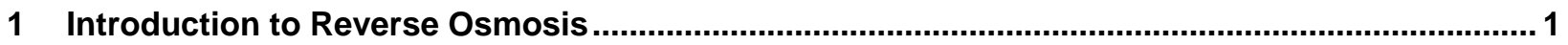

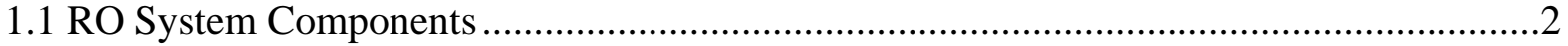

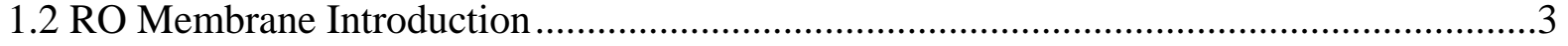

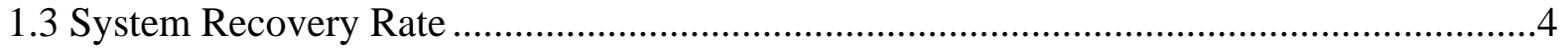

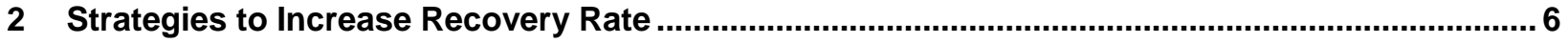

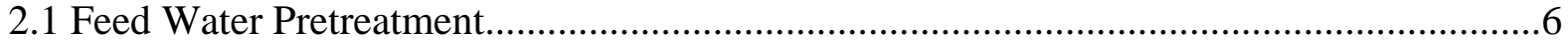

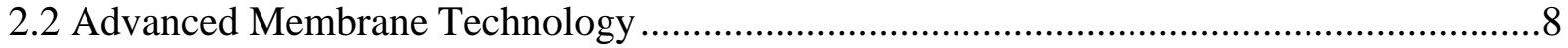

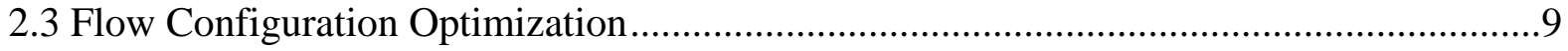

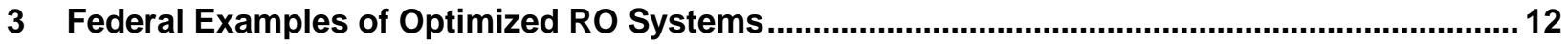

3.1 Tobyhanna Army Depot RO System Optimization ..................................................12

3.2 Sandia National Laboratories High Efficiency Reverse Osmosis System........................14

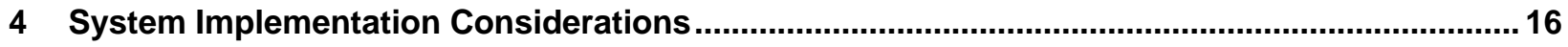

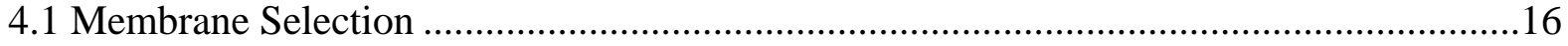

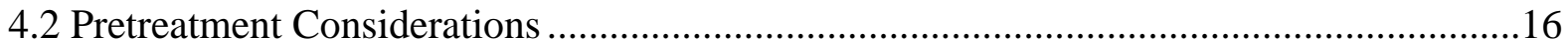

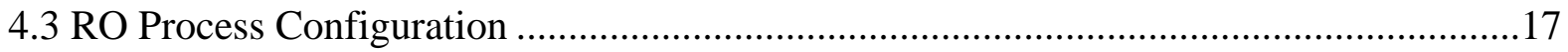

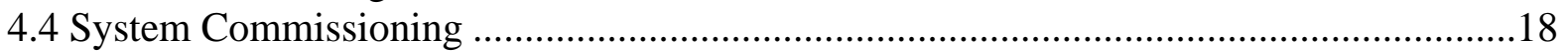

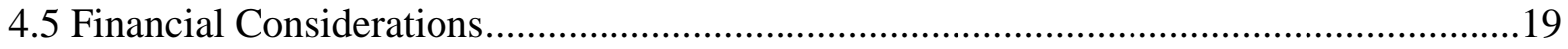




\section{List of Figures}

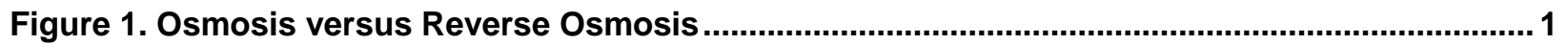

Figure 2. Diagram of an RO System with Basic Components..................................................... 2

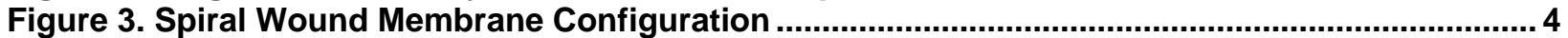

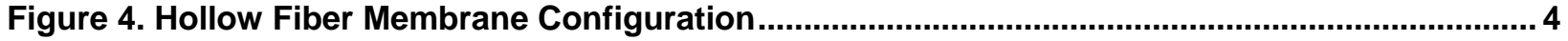

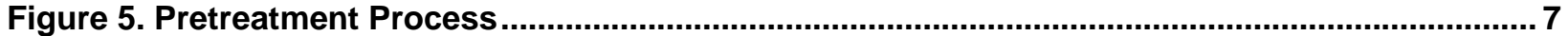

Figure 6. Single-Stage RO Flow Unit with Concentrate Recirculation ........................................... 10

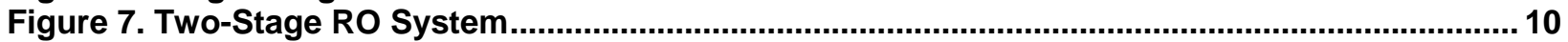

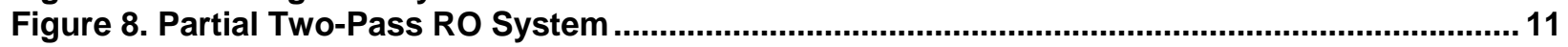

Figure 9. Tobyhanna Army Deport Two-Pass Ro System ............................................................... 12

Figure 10. Tobyhanna Army Depot RO System Optimization ........................................................... 14

Figure 11. Sandia National Laboratories HERO System Configuration ............................................ 15

\section{List of Tables}

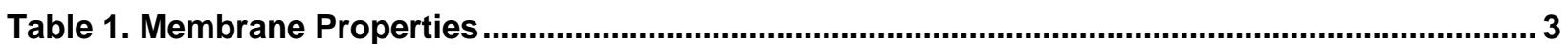

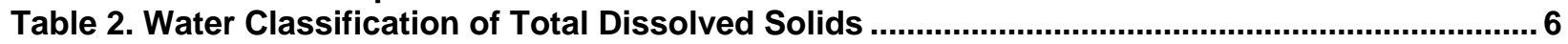

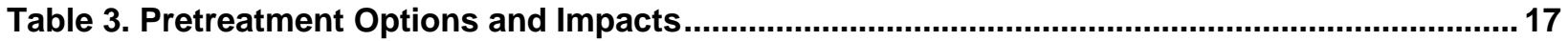




\section{Introduction to Reverse Osmosis}

Reverse osmosis (RO) technology is used in the water purification process to filter out dissolved solids and other large molecules. Typical applications of RO technology are seawater desalination, boiler feed water filtering, product rinsing, microelectronics production, laboratory testing, biotechnology, and other process that require highly purified water. There are RO systems on the market treating as little as 25 gallons per hour to more than 450,000 gallons per day.

Reverse osmosis is what the name implies: osmosis in reverse. In osmosis, water with a lower concentration of solids naturally flows through a membrane to an area of higher concentration through naturally occurring osmotic pressure, equalizing the concentration of the solute on either side of the membrane. The RO technology applies pressure to a stream of water to overcome the natural osmotic pressure. The feed water is forced through a semi-permeable membrane, emerging as purified water and leaving behind a concentrated solution of dissolved solids (see Figure 1).

Terminology: The following list provides definitions of basic terms used throughout the technology evaluation:

- Feed water: Supply water that is fed into the RO system to be treated

- Permeate: A portion of the feed water that passes through a series of membranes and is returned as purified water

- Concentrate: A portion of the feed water that is rejected by the membrane and contains the solution of impurities that have been filtered out of the permeate

- Water flux: The rate of permeate production typically expressed as the rate of water flow per unit area of membrane (e.g., gallons per square foot per day)

- Recovery rate: The ratio of permeate flow to feed water flow, which indicates the overall water efficiency of the system

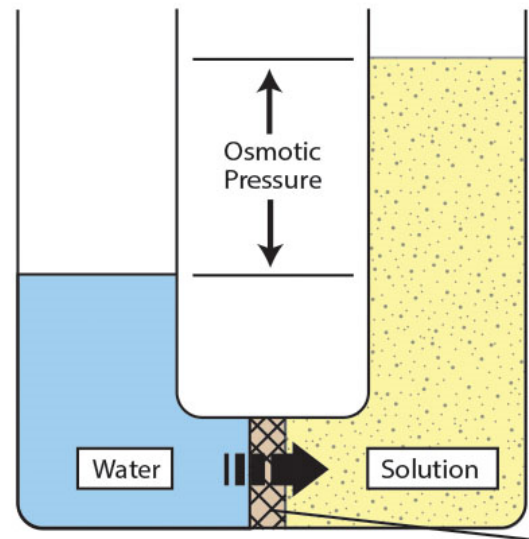

osmosis

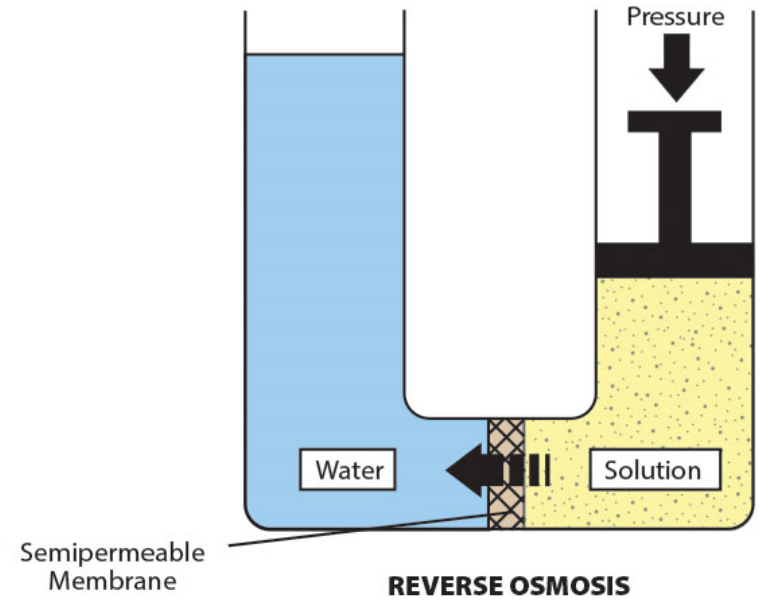

REVERSE OSMOSIS

Figure 1. Osmosis versus Reverse Osmosis 


\subsection{RO System Components}

$\mathrm{RO}$ systems consist of the following basic components, which are common to every RO system. The specifications for each component vary by application, source water quality, and the required permeate quality.

1. Pre-filter(s): It is common for pre-filters to pretreat the feed water supply before it enters an RO system. Multiple pre-filters may be used in an RO system. The most commonly used pre-filters are sediment filters used to remove sand, silt, dirt, and other sediment. Carbon filters also may be used to remove chlorine and organic compounds. (More information on pretreatment is provided in Section 2.1.)

2. Reverse osmosis membrane: The RO membrane is the heart of the system. The membrane is where the contaminants are trapped in the concentrate and purified water is produced.

3. Pressure vessels: A pressure vessel is a sealed hollow tube that houses the RO membrane elements. To force water through a semi-permeable membrane, pressure must be applied to overcome the feed water's osmotic back pressure and permeate back pressure.

4. Pumps: Pumps are required to push the water through the RO system. They must be sized to meet the required operating pressure and flow rate of the system and they constitute largest energy consuming component in the system.

5. Valves: Valves are required to control the flows and pressures of an RO system for the system to operate correctly and optimally. There are generally two valves in an RO unit on the feed water piping and on the concentrate piping.

6. Storage tank: Permeate is stored in tanks. Industrial and commercial storage tanks may hold up to 9000 gallons of water.

7. Drain line: This line runs from the outlet end of the RO membrane housing to the drain. This line is used to dispose of the concentrate rejected by the membrane element.

Figure 2 shows a schematic of an RO system with numbers corresponding to the components above.

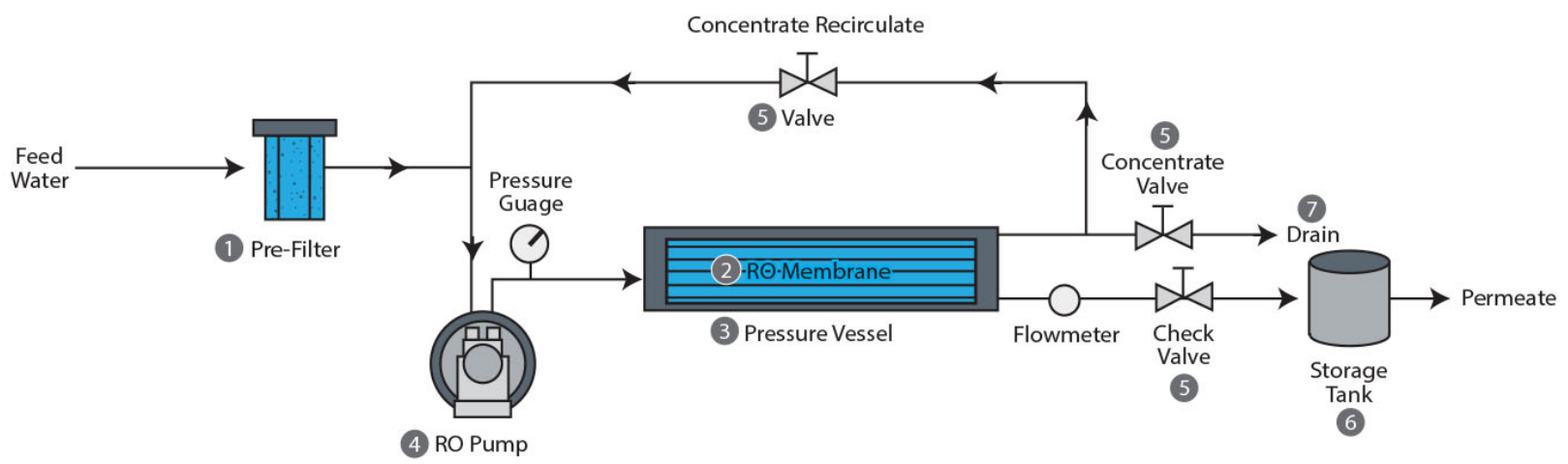

Figure 2. Diagram of an RO System with Basic Components 


\subsection{RO Membrane Introduction}

Commonly used membrane materials in RO systems are cellulose acetate (CA) membranes and thin film composite (TFC) membranes. Common membrane configurations are spiral wound and hollow fiber. The combination of membrane properties and configurations influence an RO system's effectiveness at removing impurities and passing the maximum amount of permeate through the system.

Membrane properties: RO membranes are designed for resistance to chemical and microbial damage, mechanical and structural stability over specified operating periods, and desired filtration requirements. CA membranes are tolerant to oxidizing chemicals such as chlorine but can be vulnerable to compaction (where the membrane is compressed) at high pressures, which can reduce water flux. TFC membranes are not chlorine-tolerant but can tolerate harsh chemical environments and wide ranges in water temperature and $\mathrm{pH}$, and are less vulnerable to compaction than CA membranes. TFC membranes generally have higher water flux than CA membranes because the layers are extremely thin, which creates more water transport through the membrane material. ${ }^{1}$

Depending on the feed water quality and operating conditions, membranes are changed out and disposed of several times during a system's lifetime. Generally, TFC membranes have a longer life than CA membranes because of the CA membrane's tendency to compact ${ }^{2}$.

Important properties of CA and TFC membranes are summarized in Table 1.

Table 1. Membrane Properties

\begin{tabular}{lcc}
\hline \multicolumn{1}{c}{ Feature } & $\begin{array}{c}\text { CA } \\
\text { Membranes }\end{array}$ & TFC Membranes \\
\hline Filtration of organic compounds & Low & High \\
\hline Water flux & Medium & High \\
\hline pH tolerance & $4-8$ & $2-11$ \\
\hline Temperature stability & Max $35^{\circ} \mathrm{C}$ & Max $45^{\circ} \mathrm{C}$ \\
\hline Oxidant tolerance & High & Low \\
\hline Compaction tendency & High & Low \\
Cost & Low & High \\
\hline
\end{tabular}

Membrane configurations: The two common types of membrane configuration are spiral wound and hollow fiber. In the spiral wound configuration, flat sheets of membrane are wound around the permeate collection tube to produce flow channels for permeate and feed water. Feed spacers are a netting type of material that is in between the flat sheet membrane to promote turbulent flow in the feed water, which helps the water move through the membrane, as shown in Figure 3. This design maximizes flow while minimizing the membrane module size.

\footnotetext{
${ }^{1}$ Williams, M. A Brief Review of Reverse Osmosis Membrane Technology. Williams Engineering Service Company Inc. 2003.

${ }^{2}$ FLLMTEC membranes. A Comparison of Cellulose Acetate and FILMTEC FT30 Membranes. Accessed at http://msdssearch.dow.com/PublishedLiteratureDOWCOM/dh_0062/0901b80380062296.pdf?filepath=liquidseps/pdfs/noreg/609 -00232.pdf\&fromPage=GetDoc. October 2012.
} 


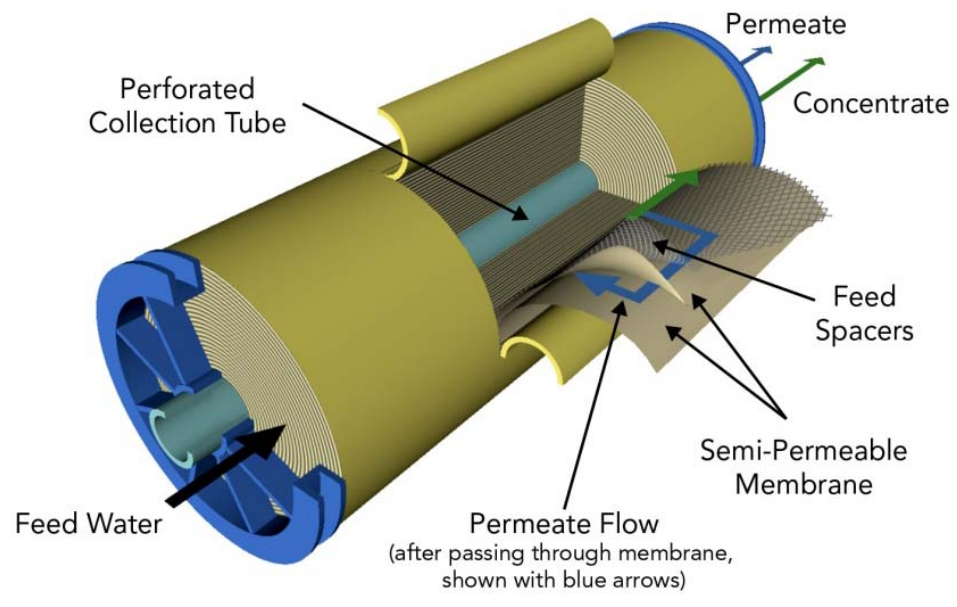

Figure 3. Spiral Wound Membrane Configuration

Hollow fiber systems are bundles of tiny, hair-like membrane tubes placed in a pressure vessel. Permeate is collected through the hollow center of the fibers. Concentrated brine is produced on the outside of the fibers contained by the module housing ${ }^{3}$ (Figure 4).

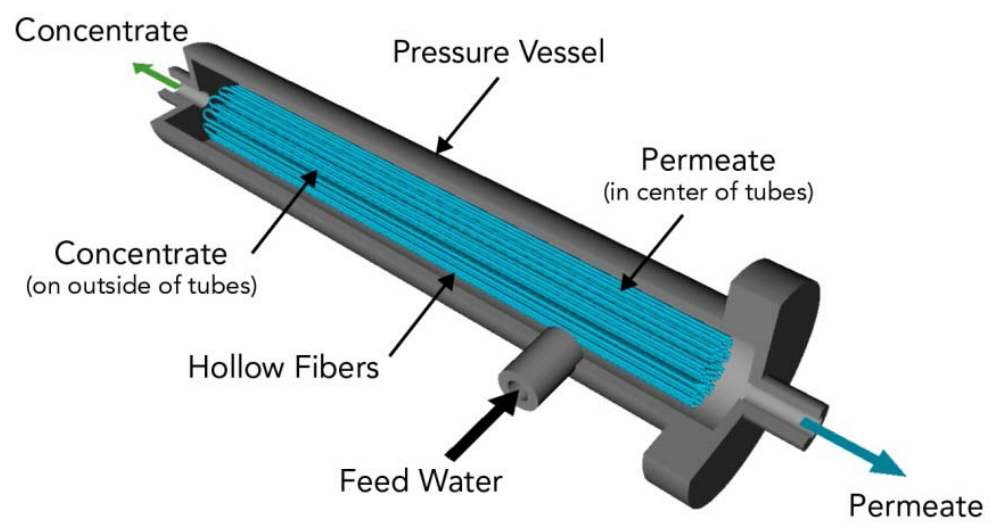

For illustrative purposes the fibers are not drawn to scale

Figure 4. Hollow Fiber Membrane Configuration

\subsection{System Recovery Rate}

The recovery rate of RO systems is the ratio of permeate flow to total feed water flow. The recovery rates of RO systems vary significantly and are influenced by the quality and constituents of the feed water, type of membrane, system configuration, and system operation. Large systems typically have recovery rates between $40 \%$ and $60 \%$. In other words, for every 10 gallons of feed water entering the system, 4 to 6 gallons of purified permeate water are produced. When systems are fully optimized, which is the focus of this technology evaluation, recovery

\footnotetext{
${ }^{3}$ General Electric. Handbook of Industrial Water Treatment, Chapter 09 - Membrane Systems. Accessed at http://www.gewater.com/handbook/index.jsp. June 2012.
} 
rates can exceed $90 \%$. The main factors that affect system recovery rate are fouling and scaling. Other factors are degradation of the system membranes and system pressure.

Fouling: Fouling occurs when suspended particles or biological compounds deposit on the membrane surfaces. Fouling of the RO membrane is heavily influenced by the quality of the incoming feed water. Membrane fouling affects the ability of the membrane to filter the feed water properly and decreases permeate production.

Scaling: Scaling is the precipitation of dissolved mineral compounds from solutions that have become saturated, producing solids trapped within the membrane. These solids can be hard to remove and decrease the overall effectiveness of the membrane, lowering the system's recovery rate.

Membrane degradation: Membranes can degrade from exposure to conditions in the system that destroys the membrane material, such as scaling, fouling, and harsh chemicals, which affects water flux and recovery rate.

Feed water pressure: Feed water pressure is another factor that affects system recovery rate. Water flux across the membrane increases in direct relationship to increases in feed water pressure given all the other factors such as osmotic pressure and water temperature are held constant. Increased feed water pressure also increases rejection of dissolved solids. The greater the water pressure within the maximum designed pressure range of the system, the better the quantity and quality of the water produced. However, a feed water pressure exceeding the designed range can cause the membranes to degrade prematurely; therefore, remaining within the design range is crucial. 


\section{Strategies to Increase Recovery Rate}

There are three basic optimization techniques discussed in this evaluation that can help to increase recovery rate and thereby reduce water use:

1. Feed water pretreatment

2. Advanced membrane technologies

3. Flow configuration optimization

These techniques can be applied individually or together when designing or retrofitting an RO system.

\subsection{Feed Water Pretreatment}

Pretreatment of the feed water entering an existing RO system can minimize fouling of the membrane and thereby increase the overall recovery rate of the system. The feed water, depending on its source, may contain various concentrations of suspended solids and dissolved matter, including both organic and inorganic substances. Suspended particles can settle on the membrane surface, blocking feed channels and increasing friction losses across the system. Dissolved solids can precipitate out of the solution and cause scaling. Pretreated water entering the RO system can reduce the amount of work of the RO pump, thus reducing energy consumption.

Common dissolved solids are sodium, calcium, potassium, phosphates, and nitrates. These particles typically cannot be mechanically filtered with a pore size smaller than 2 microns. The measure of combined inorganic and organic dissolved solids in water is called total dissolved solids (TDS). Table 2 provides TDS for specific water classifications.

Table 2. Water Classification of Total Dissolved Solids

\begin{tabular}{lc}
\hline \multicolumn{1}{c}{ Water type } & TDS (mg/L) \\
\hline Potable water & $<500$ \\
Freshwater (not treated) & $<1500$ \\
Brackish water & $1500-5000$ \\
Saline water & $>5000$ \\
\hline
\end{tabular}

Suspended solids are particles suspended in solution and large enough to be trapped by a 2 micron filter. Examples include silt, industrial byproducts, and decaying plant matter. The measurement of suspended solids is total suspended solids (TSS).

Various pretreatment technologies can reduce both TDS and TSS in the feed water, allowing the RO membrane to increase the permeate production. The overall influence on recovery rate with pretreatment system varies significantly depending on the constituents in the feed water, type of RO membrane, and operational practices.

A typical pretreatment process includes the following steps:

1. Removal of large particles (typically larger than 5 microns) with a mesh strainer or traveling screen 
2. Disinfection to kill biological growth

3. Coagulant or flocculants to conglomerate particles that can be filtered out

4. Chemical treatment to inhibit scaling

5. Activated carbon filter to remove chlorine and organic compounds

6. Final removal of suspended solids using cartridge filter

The flow diagram of a typical pretreatment system for surface water is shown in Figure 5.

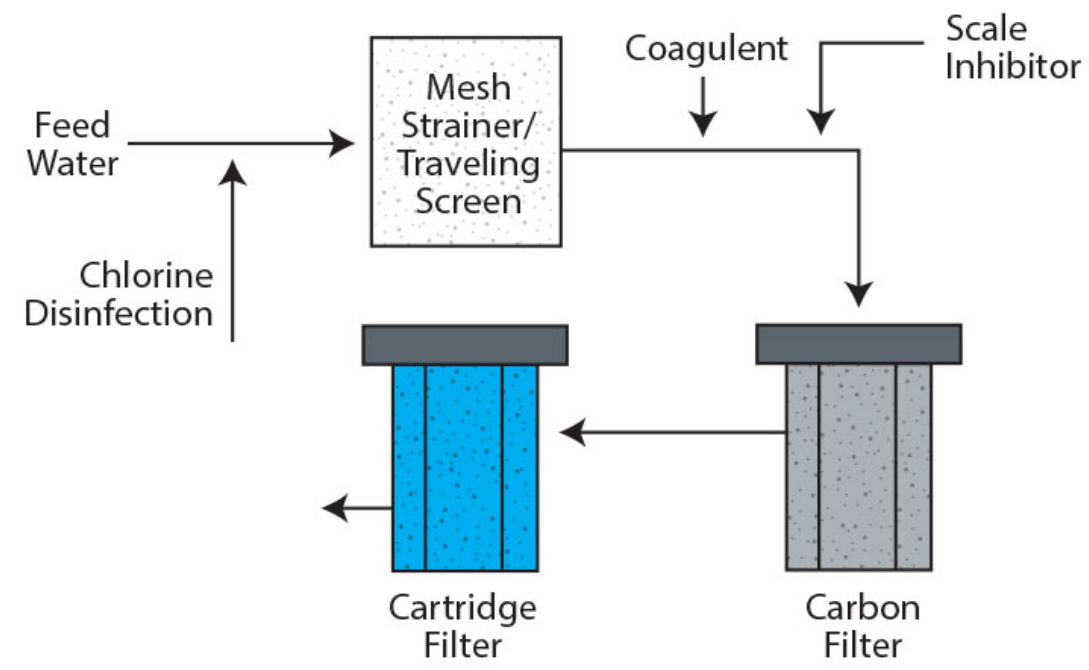

Figure 5. Pretreatment Process

Depending on the source of the feed water, the initial removal of large particles from the feed water is accomplished using mesh strainers or traveling screens. Mesh strainers are used in well water supply systems to stop and remove sand particles that may be pumped from the well. Traveling screens are used mainly for surface water sources, which typically have large concentrations of biological debris.

The surface feed water is usually disinfected after the screen to control biological activity. Biological activity in well water is typically low, and in majority of cases, well water does not require chlorination. Addition of flocculants or coagulants neutralizes surface charges of colloidal particles so that suspended particles can aggregate and be removed via filtration. Well water usually contains low concentrations of suspended particles, due to the filtration effect of the aquifer, therefore flocculants and coagulants may not be necessary. In addition, for both feed water sources, it is common for pretreatment systems to use scale-reducing chemicals such as lime, which increases the water's $\mathrm{pH}$ that precipitates out the formation of calcium carbonate and magnesium hydroxide particles. Lime clarification reduces hardness and alkalinity.

After passing through a scale inhibitor, some surface water may still contain high concentrations of dissolved organics in the feed water. Those can be removed by passing feed water through an activated carbon filter. The use of cartridge filters provides a final barrier to water-borne 
particles before the water enters the RO membrane. Cartridge filters commonly used in RO applications are rated for removal of particles as small as 1 micron. ${ }^{4}$

Advanced filtration technology, such as microfiltration, ultrafiltration, and nanofiltration, can also be used as pretreatment in RO systems. Microfiltration removes particles larger than 0.1 micron; ultrafiltration removes particles larger than 0.01 micron, and nanofiltration removes particles larger than 0.001 micron. Microfiltration can remove suspended solids and bacteria, ultrafiltration can filter viruses, and nanofiltration can remove organic matter and some dissolved solids. ${ }^{5}$ These advanced systems can provide better feed water quality than other conventional pretreatment steps; however, the cost of this equipment is still high in comparison.

\subsection{Advanced Membrane Technology}

There are several new advanced membrane technologies for RO systems to assist in optimal membrane selection for specific applications. Advances in membrane technology allow more options for higher water production rate. These advances include larger active surface areas, higher permeable membranes, larger diameter spiral wound elements, and low fouling membranes. These new features, if designed and implemented properly, can reduce energy consumption and increase water recovery rates of the systems.

Larger active surface area: Spiral wound membranes with a large active surface area are commercially available. These types of membranes enlarge the elements' filtration area without increasing the elements' physical size. For example, a circa 1980 filter with an area of $320 \mathrm{ft}^{2}$ can now have a filtration area of $440 \mathrm{ft}^{2}$ with new spiral wound membranes. This increase in area translates into a $38 \%$ increase in water production from the same pressure vessel. ${ }^{6}$

Higher permeability: Membranes with high permeability incorporate nano-particles within TFC membranes. Nano-particles are encapsulated in the conventional RO membranes, which change the structure of the thin-film surface, allowing more water to pass through while meeting a high rejection rate. This type of membrane requires lower operating pressure, which reduces energy requirements and also increases water flux and recovery rate. The membranes can cost slightly more than conventional units; however, higher permeability can decrease operating costs because of energy and water savings.

Larger diameter spiral wound elements: For large scale RO systems, large diameter spiral wound modules enable significant reductions in RO plant capital cost and life-cycle cost. ${ }^{7}$ The standard size for spiral wound elements in industrial RO systems is 8 inches. However, within large scale RO plants, 8-inch diameter membranes are not the most efficient choice because of the number of elements, pressure vessels, piping, and connections that must increase in direct proportion to the increase in flow capacity. The optimum size for large scale systems is 16-inch diameter spiral wound elements, which allows the membrane's active area and productivity to increase to 4 times that of standard RO modules. For typical brackish water RO systems, use of

\footnotetext{
${ }^{4}$ Hydranautics. Pretreatment. Accessed at http://membranes.com/docs/trc/pretreat.pdf. October 2012.

${ }^{5}$ Safe Drinking Water Foundation. Ultrafiltration, Nanofiltration, and Reverse Osmosis. Accessed at http://safewater.org/. October 2012.

${ }^{6}$ Bartels C., M. Hirose, H. Fujioka. Performance Advancement in the Spiral Wound RO/NF Element Design, Oceanside, CA. 2007.

${ }^{7}$ Hallan M.J., J.E. Johnson, M.S. Koreltz, M.H. Perry. Design, Development, and Evaluation of Sixteen Inch Diameter RO Modules. IDA World Congress. Maspalomas, Spain. 2007.
} 
16-inch elements would save approximately $15 \%$ in material costs on the membrane racks. ${ }^{8}$ However, cost savings and productivity increases tend to be project specific and need to be evaluated case by case.

Low-biofouling feed spacers: Feed spacers are the material responsible for maintaining channels of water flow between the two faces of the membrane in a spiral wound element. Feed spacers can foul easily if feed water has a large amount suspended solids that plug the channels and reduce the flow through the membrane. In contrast, low-biofouling feed spacers have a larger open cross-sectional area than traditional spacers, reducing pressure drop and allowing more effective cleaning, and thereby reducing fouling. Low-biofouling feed spacers minimize pressure drop across the element, which increases water productivity. ${ }^{9}$

\subsection{Flow Configuration Optimization}

There are different types of flow configurations in RO systems including single-stage, two-stage, single-pass and two-pass systems. RO systems can contain one to several groups of pressure vessels. Each group of pressure vessels is called a stage. RO systems can also have distinct RO units, referred to as a pass. For applications where high purity water is required, two-pass systems are common because they essentially treat the water twice in two distinct systems. These flow configurations can be optimized, influencing overall system performance.

In the following sections, flow configuration options that allow higher water recovery rates are described.

Concentrate recirculation: The simplest membrane element assembly is called a single-stage system that typically sends the concentrate directly to drain. Such a configuration operates at a limited recovery rate. To increase the overall system recovery rate, a portion of the concentrate stream is returned to the supply and blended with the feed water. The concentrate recycling configuration is shown in Figure 6. The potential disadvantage of concentrate recirculation design is related to the possible need for a larger feed pump to handle higher feed flow. In this case, energy use would increase for the system. Because the feed water blends with the concentrate stream, the average feed salinity can change. Therefore, if a system is converted to a concentrate recirculation system, the feed water must be reevaluated for pretreatment methods (as discussed in Section 2.1). Figure 6 shows an example schematic of a concentrate recirculation process in a single-stage RO system.

\footnotetext{
${ }^{8}$ Koch Membrane Systems. Membrane Datasheets. Accessed at http://www.kochmembrane.com/. April 2012.

${ }^{9}$ Bartels, C., M. Hirose, H. Fujioka. Performance Advancement in the Spiral Wound RO/NF Element Design, Oceanside, CA. 2007.
} 


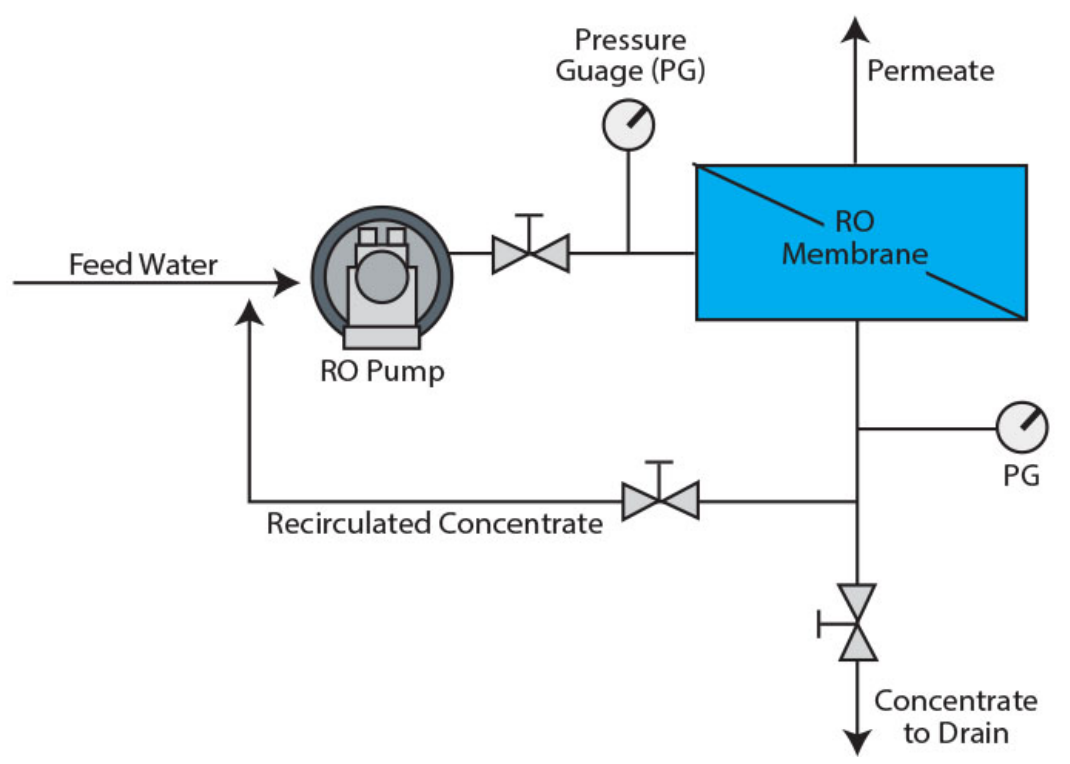

Figure 6. Single-Stage RO Flow Unit with Concentrate Recirculation

Concentrate staging: Commercial RO units usually consist of a multistage array of pressure vessels. Two-stage configuration is the most common configuration. In each stage, pressure vessels are connected in parallel, with respect to the direction of the feed and concentrate flow. The objective of the pressure vessel configuration is to maintain a similar flow rate per vessel through the length of the system and to maintain flow within the limits specified for a given type of membrane element.

A simplified block diagram of a two-stage RO unit is shown in Figure 7. The concentrate from the first stage becomes the feed to the second stage; this is what is meant by the term “concentrate staging.” Flow configurations that utilize concentrate staging produce more highly purified permeate and increase the permeate recovery rate since the concentrate from the first stage is filtered again in the second stage instead of being rejected.

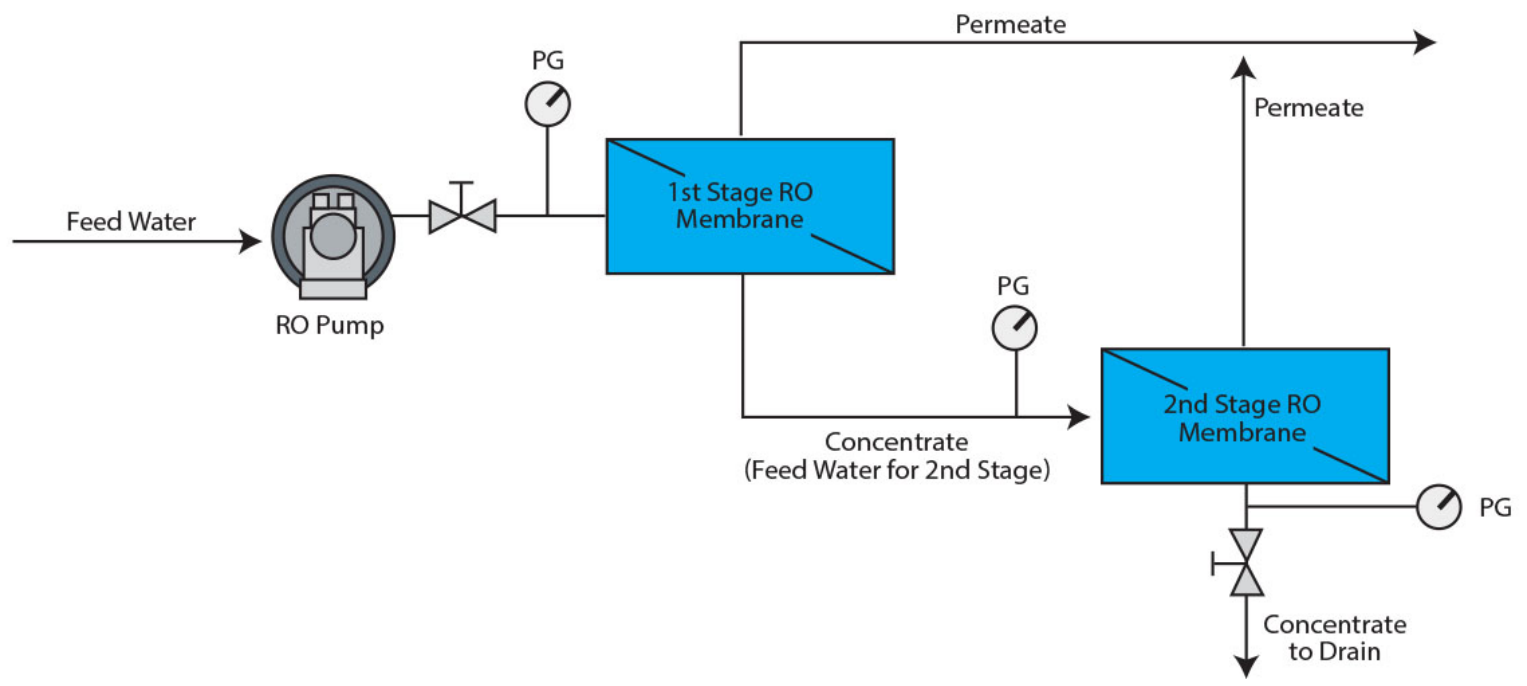

Figure 7. Two-Stage RO System 
Permeate staging: For some applications, the single-pass RO system may not be capable of producing permeate water to a required quality. To achieve an additional reduction in permeate salinity, the permeate water produced in the first pass is filtered again in a second RO system. This configuration is called a two-pass design, or "permeate staging." Depending on quality requirements, all or part of the first-pass permeate volume is filtered again in the second-pass system. The system configuration is known as a complete or partial two-pass system depending on whether all of the permeate is fed to the second pass.

A partial two-pass system, shown in Figure 8, splits the permeate from the first pass into two streams. One stream is processed by the second-pass unit, and is then combined with the unprocessed part of the permeate from the first pass. Provided that the partial second-pass system can produce the required permeate quality, this configuration has smaller capital and operating costs and a higher combined permeate recovery rate compared to a complete two-pass system. Two-pass systems commonly recirculate concentrate into the first-pass unit. The dissolved solids concentration in the concentrate from the second pass is usually lower than the concentration of the feed water. Therefore, blending feed water with the second-pass concentrate slightly reduces the salinity of the feed water and increases the overall utilization of the feed water.

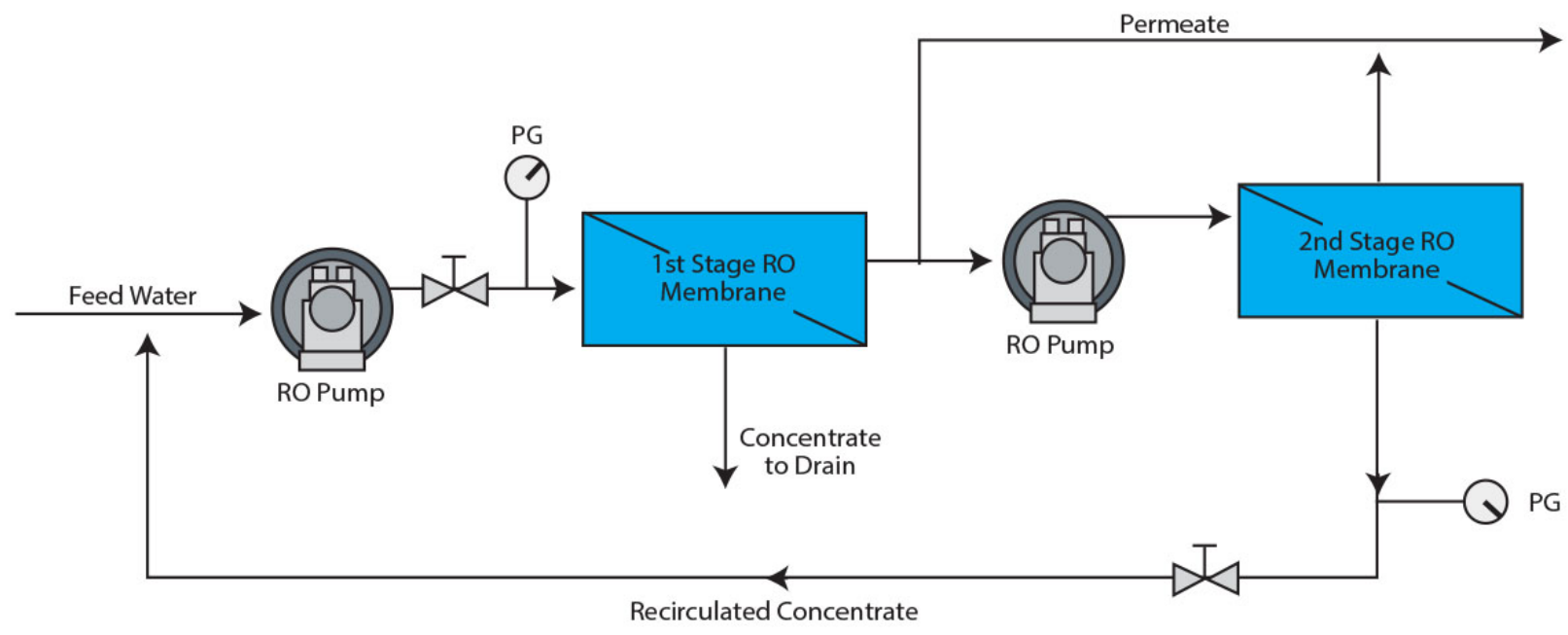

Figure 8. Partial Two-Pass RO System 


\section{Federal Examples of Optimized RO Systems}

To help illustrate techniques for optimizing RO systems, the following section provides two examples of RO system optimization at Federal sites: Tobyhanna Army Depot and Sandia National Laboratories.

\subsection{Tobyhanna Army Depot RO System Optimization ${ }^{10}$}

Tobyhanna Army Depot, a major facility of the U.S. Army Communications-Electronics Command, is the largest full-service electronics maintenance facility in the Department of Defense. Tobyhanna Army Depot houses the Industrial Operations Facility, which contains industrial metal finishing, plating, painting, sandblasting, and industrial wastewater treatment operations and equipment. This facility has a large RO system that provides purified water for metal finishing and electroplating operations. The system is a two-pass design, with each pass containing three RO membrane stages. The two-pass design treats the concentrate twice and recycles it back within the system. Both passes are three-stage systems with varying parallel pressure vessel configurations, as shown in Figure 9. The first-pass RO produces purified permeate that is sent to the second-pass RO to be purified further. Typically, the first-pass RO reduces TDS by $80 \%$ to $85 \%$. The second-pass RO accomplishes additional TDS reduction to over $98 \%$ to $99.5 \%$.

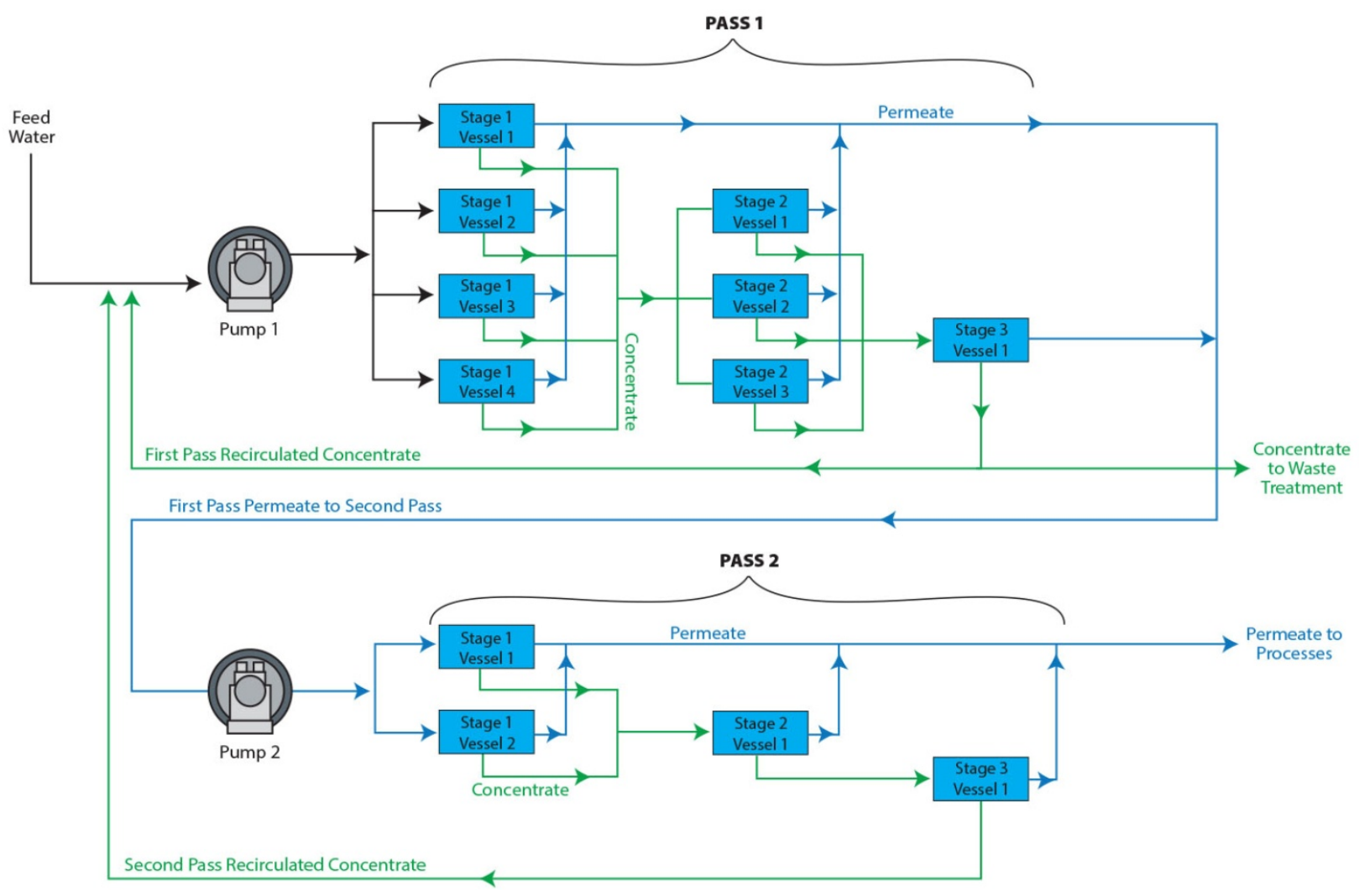

Figure 9. Tobyhanna Army Deport Two-Pass RO System

\footnotetext{
${ }^{10}$ Information presented on Tobyhanna Army Depot reverse osmosis system and optimization program was provided directly by engineering staff in October 2012.
} 
Soon after construction and initial operation of the system, it was discovered that the commissioning process had not adequately balanced the required pressures and flow rates necessary for stable operations. This imbalance caused excessive membrane flux within the firststage pressure vessels, while limiting the necessary membrane flux throughout the majority of the remaining pressure vessels. Because the system could not achieve the necessary flux in the second stage, the system required an increase in the trans-membrane pressure in order to force more water through the membrane. The system required 350 - 360 pounds per square inch (psi) to achieve a flow rate of $50-60$ gallon per minute (gpm). The increase in system pressure thus increased the power requirements of the system.

The investigation also determined that the original two-pass design recycled concentrate streams from both the first pass and second pass directly back to the first-stage RO feed pump with no pre-filtering, causing carbonate based solids to precipitate in the system, fouling the membrane surfaces. Finally, the investigation determined that the RO feed water contained significant levels of naturally occurring organic material, dissolved and precipitated solids, and biological matter, all of which contributed to membrane fouling.

Once fouled, the available effective membrane surface area was significantly reduced, inevitably decreasing permeate production relative to the design specification. To restore appropriate permeate production, RO membrane elements required extensive weekly or bi-weekly chemical cleaning. This frequent cleaning and oxidizing compounds such as chlorine deteriorated the membranes, which had to be replaced every 6 months. The total cost associated with cleaning and replacing RO membranes totaled over $\$ 100,000$ annually.

Because of these extensive problems, Tobyhanna Army Depot engineers conducted a comprehensive technical review of the system. It was concluded that the root of the system problems was inadequate pressure balancing and lack of pretreatment of the RO feed water. An improvement plan was formulated to optimize the system.

The process improvements include:

- Microfiltration before the RO membranes to remove precipitated and insoluble elements within the feed water, including natural organic matter and synthetic surfactants

- Control of biological growth with the introduction of oxidizers (sodium hypochlorite and peroxide) into the feed water at controlled $\mathrm{pH}$

- Dechlorination prior to introducing water to the RO unit to prevent chemical attack of the RO membranes

- Balancing of the pressures throughout the RO equipment to maintain manufacturerrecommended concentrate flow rates, pressure drops, and water flux

- Pretreatment and balancing of the system reduced the hydraulic resistance of the system that resulted in maximizing flux and minimizing membrane pressure, allowing a flow rate of 100 - 125 gpm with $300-320$ psi feed water pressure, which increased the flow rate by $100 \%$ at a lower operating pressure and reduced energy requirements of the system 
- Monitoring and control of feed water dissolved solids concentrations via conductivity measurements to minimize feed water osmotic pressure and to maximize flux of water through the membrane

- Modification of the RO unit configuration that recycles the first and second pass concentrate streams back to the RO feed tank for proper pre-filtering and $\mathrm{pH}$ adjustment; this resulted in reduced solids concentration and improved feed water $\mathrm{pH}$ control while simultaneously allowing for additional permeate recovery

The optimized system configuration is shown in Figure 10.

Tobyhanna Army Depot experienced impressive savings as a direct result of these improvements to the system. The recirculation of the concentrate streams satisfies the bulk of the demand, annually totaling 27 million gallons (Mgal). As little as $3.5 \mathrm{Mgal}$ per year of fresh potable water is needed to replenish unrecoverable evaporative losses, and losses due to effluent discharges. The system design changes reduced the osmotic pressure, which in turn increased the system flux, allowing the system to produce $150 \%$ of the designed permeate output of the system. The excess permeate is used in the air scrubbers, which further reduces demand on potable water. Reduction of fouling issues has extended the life of the RO membranes to nearly 5 years and only requires one annual cleaning, resulting in an annual cost savings of over $90 \%$.

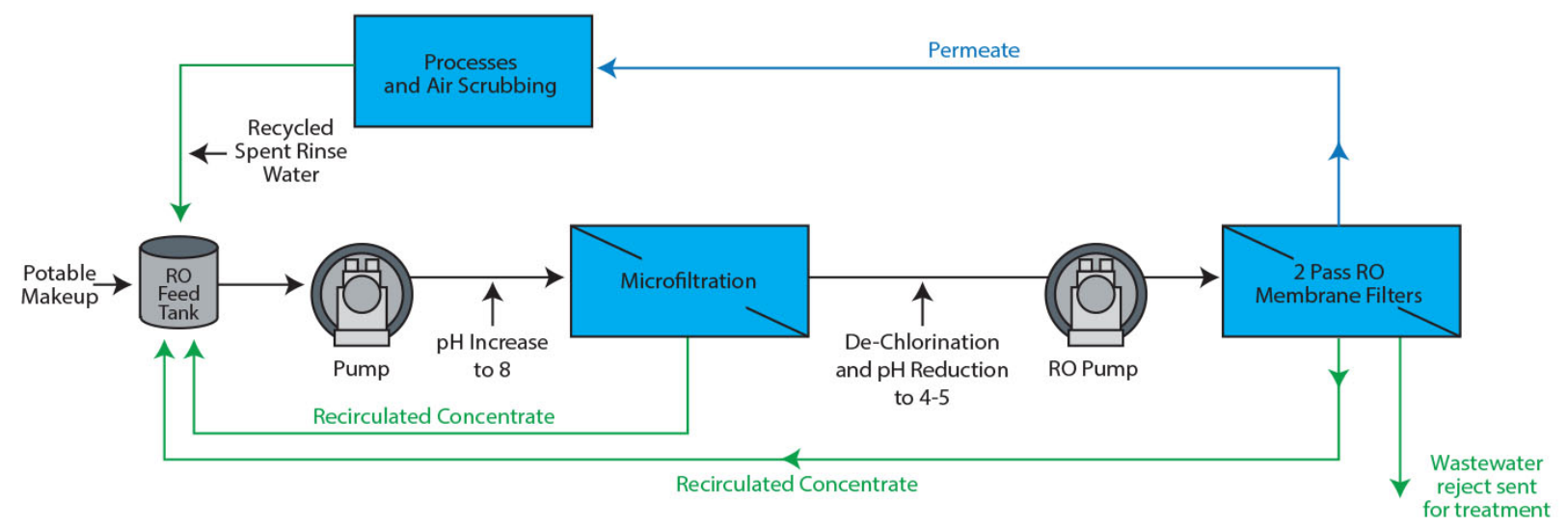

Figure 10. Tobyhanna Army Depot RO System Optimization

\subsection{Sandia National Laboratories High Efficiency Reverse Osmosis System}

Sandia National Laboratories in Albuquerque, New Mexico, upgraded the RO system at the Microsystems and Engineering Sciences Applications (MESA) complex to a High Efficiency Reverse Osmosis $\left(\mathrm{HERO}^{\mathrm{TM}}\right.$ ) system in 2005. The MESA complex houses microelectronics processing that requires highly purified water for microelectronics rinsing.

HERO technology is a proprietary pretreatment system that improves the recovery rate of RO systems. HERO is specifically designed to purify difficult to treat feed waters, which can often pose problems to a conventional RO process. HERO in general produces purer water than conventional RO, and the water flux (gallons per square foot of membrane per day) for HERO is nearly double that of conventional RO.

The HERO system employs a three-step process: 
1. Removal of dissolved solids (such as calcium and magnesium) by increasing the alkalinity of the feed water, which creates a cation exchange where hydrogen ions are exchanged with hardness ions, reducing the hardness of the water

2. Degasification that removes carbon dioxide gas created in the cation exchange process

3. Acidification that decreases the $\mathrm{pH}$ to increase solubility of silica and destroy biological growth

HERO system RO membranes are operated in a high $\mathrm{pH}$ environment that limits the potential foulants and substantially improves overall performance, producing higher quality permeate. An additional benefit can be the elimination of additional anti-scaling chemicals because the pretreatment process reduces hardness in the feed water, which in turn can reduce the cleaning and maintenance requirements compared to conventional RO systems. For small systems, the capital costs for HERO are higher than conventional systems due to the higher cost of pretreatment. However, for systems larger than 50 gpm, capital costs for HERO are generally about $15 \%$ lower because of the higher flux rate, which can reduce the number RO membranes required. HERO typically has lower energy cost as well. ${ }^{11}$

For Sandia National Laboratories, the HERO system has created a recovery rate of 95\%. Annually, Sandia National Laboratories saves approximately 34 Mgal gallons of water. ${ }^{12}$ Sandia National Laboratories also recycles approximately $6 \mathrm{Mgal}$ of the spent rinse water from the MESA processes in the HERO system. In addition to recycling, spent rinse water is also reclaimed for other applications. Spent rinse water is reclaimed for use in the acid waste scrubbers, which remove contaminants from exhaust of the microelectronics process. Rinse water is also reclaimed for make-up for nearby cooling towers (Figure 11). In total, $40 \mathrm{Mgal}$ of water is reclaimed annually in both the acid waste scrubbers and cooling towers.

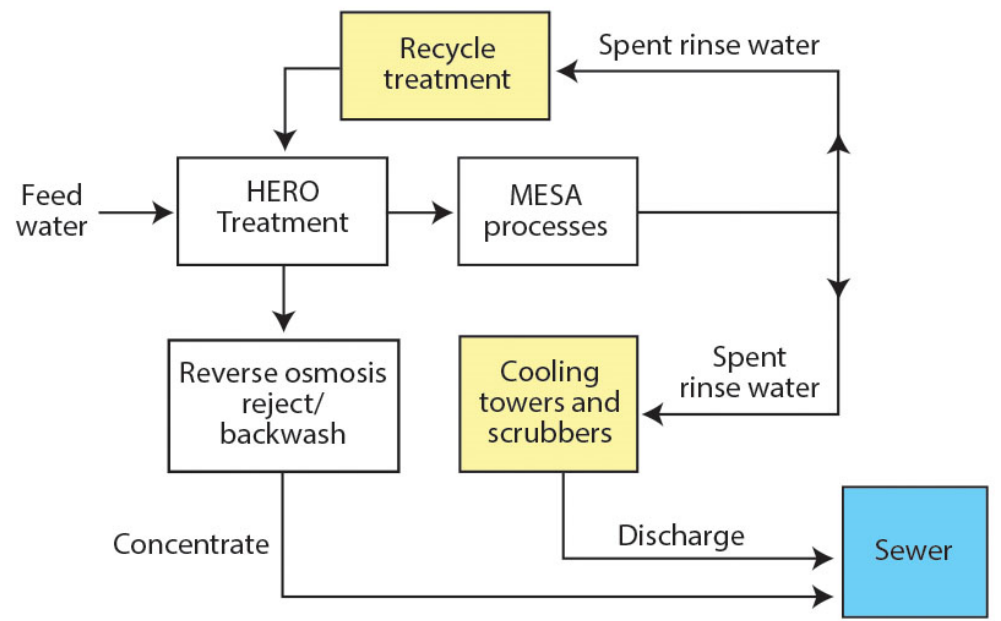

Figure 11. Sandia National Laboratories HERO System Configuration

\footnotetext{
${ }^{11}$ Federal Energy Management Program. Saving Energy, Water, and Money with Efficient Water Treatment Technologies. U.S. Department of Energy. Washington, DC. 2004.

${ }^{12}$ Federal Energy Management Program. Microelectronics Plant Water Efficiency Improvements at Sandia National Laboratories. U.S. Department of Energy. Washington, DC. 2009.
} 


\section{System Implementation Considerations}

As with any system, the success of RO system operation depends on how the components are specified, installed and operated. As touched on in previous sections, some of the key parameters that are important to focus on include membrane selection, pretreatment options, RO process configuration, system commissioning, and financial considerations. These criteria can be reevaluated during the RO system's lifetime and adjusted based on revised feed water conditions and permeate requirements. Some best practices are provided below for reference.

\subsection{Membrane Selection}

Compare the specific operating conditions of the current system to the specification of the membrane to make sure that the membrane is suitable for the application. Consider the following characteristics:

- Membrane fouling rates

- Water flux specification

- Solid rejection rate

- System pressure requirements

- Membrane response to cleaning operations and tolerance of cleaning procedures

- Tolerance of $\mathrm{pH}$

- Temperature range

- Chemical abrasion resistance

Such information provides a good indication of the suitability of a particular membrane for an application. However, the specification is based on the test results generated under artificial laboratory testing conditions, which are limited in projecting the membrane's performance under real world conditions. Therefore, to ensure an optimized design and operation of an RO system for a given application, these key characteristics must be reevaluated once the application is operating to ensure that the membrane is performing as required.

There are many commercially available RO membranes, both of the CA and TFC type. Advanced membranes are also available that produce larger surface area, high permeability, and reduced fouling. In the United States, main RO membrane manufacturers include but are not limited to Filmtect (DOW), Hydranaustics, GE Osmonics, GE Osmonics/Desal, Koch Membrane System. Inc, Trisep, and Torary. These membrane suppliers provide detailed technical specifications of their membrane elements.

\subsection{Pretreatment Considerations}

When selecting the pretreatment system, it is imperative to thoroughly understand the quality of the supply feed water. It is recommended that a comprehensive water analysis be performed that provides the type and concentration of all major constituents, including:

- Dissolved solids (TDS) 
- Organic compounds

- Suspended solids (TSS)

- Turbidity

- $\mathrm{pH}$

This information will help to ensure that an appropriate pretreatment system is designed that includes filtration processes that handle all of the major issues that can foul and scale the RO membrane and reduce recovery rate. Based on information provided in Section 2.1, Table 3 summarizes the pretreatment options that can help to limit negative effects on the RO membrane.

Table 3. Pretreatment Options and Impacts

\begin{tabular}{lccc}
\hline Pretreatment Option & $\begin{array}{c}\text { Reduces } \\
\text { Dissolved } \\
\text { Solids }\end{array}$ & $\begin{array}{c}\text { Reduces } \\
\text { Suspended } \\
\text { Solids }\end{array}$ & $\begin{array}{c}\text { Limits } \\
\text { Biological } \\
\text { Growth }\end{array}$ \\
\hline Coarse/mesh strainer & & $\bullet$ & $\bullet$ \\
Disinfection & & & \\
$\begin{array}{l}\text { Chemical treatment } \\
\text { (e.g., lime) }\end{array}$ & - & & \\
Acidification & $\bullet$ & $\bullet$ & \\
Coagulation/flocculation & & $\bullet$ & $\bullet$ \\
Cartridge filter & & & $\bullet$ \\
Activated carbon filter & $\bullet$ & $\bullet$ & $\bullet$ \\
Microfiltration & & $\bullet$ & \\
Ultrafiltration & & & \\
Nanofiltration & $\bullet$ & & \\
\hline
\end{tabular}

\subsection{RO Process Configuration}

The configuration of RO systems can influence the system's recovery rate significantly. The basic configuration types are a single-stage, multistage, and two-pass process units that can be combined to produce plants of various configurations. Optimization of these process configurations can yield efficiency improvements.

Multistage configuration: Multistage RO systems produce higher water recovery rates than single-stage systems. Instead of the concentrate being sent to the drain, the concentrate passes through additional membrane elements that generate more permeate from the same volume of total feed water entering the system. An important consideration when optimizing multistage RO systems for high recovery rates is properly setting system pressure and flow requirements within the manufacturer's specifications. Ensure that the membrane elements, pressure vessels, pressure pumps, and piping configuration are adequately designed to meet the pressure and flow requirements. Additional pumps may be needed, which will add to the energy consumption of the system. Also, disposal or treatment of the concentrate from multistage systems may be different than single-stage systems because the concentrate will have higher levels of dissolved solids. These added costs should be considered when evaluating the potential water reduction.

Two-pass configuration: The use of a two-pass membrane process configuration can be necessary when the target permeate production cannot be accomplished in a single pass. 
Compared to single-pass systems, two-pass systems tend to have lower water recovery rates and higher energy consumption because the permeate from the first pass is further filtered by the RO membranes in the second pass, and therefore only part of the permeate from the first pass becomes the final permeate of the system. An option to increase the recovery rate is to convert a full two-pass configuration to a partial two-pass configuration so that only part of the permeate from the first pass will go through the second pass. The system permeate is the combination of the permeate from the first pass and the permeate from the second pass. The key factor to consider for this optimization is that the system permeate must be carefully analyzed to ensure that the overall permeate still meets the purity requirement. Another optimization option to achieve higher water recovery rate for two-pass systems is to recycle the concentrate from the second-pass feed to the first-pass feed. Since the feed water of the second pass is the permeate of the first pass, it has relatively high purity and therefore the concentrate from the second pass tends to have higher purity than the feed water.

Commercially available RO process design software, such as ROSA ${ }^{\circledR}$ from FILMTEC ${ }^{\text {TM }}$ and IMDDdesign $^{\circledR}$ from Hydranautics ${ }^{\mathrm{TM}}$, can be used to optimize the configuration of RO systems and optimize membrane selection and operation. These software tools require the user to enter information on the current RO system design and allow the user to test multiple RO configurations with different membranes to ascertain the best design for the application.

\subsection{System Commissioning}

RO systems should always be commissioned during system startup to ensure that the components are operating to the design specifications and meeting the expected permeate recovery rates. Additionally, a continuous commissioning process should be in place to monitor and track performance during the daily operation of the system. Continuous commissioning needs to focus not only on contaminant removal and membrane function, but also on other operational parameters of the system.

Pressure, temperature, $\mathrm{pH}$, and flow rates are the key indicators of system function. The control points in system that should be focused on are:

- Feed water temperature

- Valve positions

- $\quad$ Pressure set points

- Pump operation

In addition, balancing pressures to maintain manufacturer recommended pressure drops will help to achieve the desired permeate recovery rate. It is also important to configure the system keeping in mind the desired flow rate through the pressure vessels. Very high flow through a pressure vessel will result in a high pressure drop and possible structural damage of the element. Very low flow may result in excessive buildup of solids at the membrane surface and cause fouling, decreasing the recovery rate. ${ }^{13}$

\footnotetext{
${ }^{13}$ Hydranautics. Flow Configuration. Accessed at http://www.membranes.com/docs/trc/flowcon.pdf. June 2012.
} 
Once the RO system has been balanced and commissioned, continuous monitoring of the key indicators will provide the operations team with baseline information system function. The operations team can then more quickly identify problems when points start to drift, thereby keeping the recovery rate at the desired level the majority of the time.

\subsection{Financial Considerations}

The cost of optimizing an RO system is influenced by many parameters that are specific to the application and operation of the system, such as feed water quality, membrane type, system configuration, and purity requirements. Therefore, to determine the costs and financial benefits of optimization options, the financial analysis must take into account the site-specific nature of the technology. Costs to consider in a financial analysis include:

- Analysis cost to determine system optimization options

- Pretreatment capital equipment and installation

- Plumbing and electrical capital equipment and installation

- Operation and maintenance of the system including commissioning

- Chemicals

- Energy used to power the systems

These costs should be compared to the benefits resulting in the optimization to determine the overall cost effectiveness. With the increased recovery rate, less feed water will be needed to meet the required quantity of permeate production, which will reduce costs. Wastewater treatment costs also may decline if discharged concentrate is reduced.

Another savings stream to consider is in membrane maintenance. Pretreatment optimization plays a key role in keeping the membrane clean and efficient, which may reduce the frequency of membrane cleaning and extend the life of the membrane elements. Lastly, optimization of membrane elements and system configuration might reduce operating pressure. When the system uses lower operating pressure, less energy is consumed, resulting in reduced energy cost for the system.

The Bureau of Reclamation of the U.S. Department of Interior developed a detailed cost evaluation software tool for RO process configuration, called WTCost ${ }^{\odot}$ software. ${ }^{14}$ This application estimates and compares the cost of water treatment technologies based on local water analysis and desired capacity. Users input the plant parameters such as cost of electricity, labor, water, materials, and other applicable variables required for the system. The software provides default values for all variables.

Because of the site specific nature of large RO systems, there is not a typical range of potential energy, water, and cost savings from system optimization. However, optimizing large RO systems often results in significant cost savings that outweigh the upfront costs, as presented in the Tobyhanna Army Depot and Sandia National Laboratories case studies.

\footnotetext{
${ }^{14}$ Moch. I., W.R. Querns, and D. Steward. A Computerized Water Treatment Cost Estimating Program. Boulder, 2004.
} 